\title{
APPOINTMENTS AVAILABLE FOR POSTGRADUATE TRAINING IN ANAESTHESIA IN CANADIAN HOSPITALS APPROVED BY THE ROYAL COLLEGE OF PHYSICIANS AND SURGEONS OF CANADA
}

THE following information is derived from a survey recently conducted by the Canadian Anaesthetssts' Society, and is published as provided by the hospitals and university departments concerned It will be noted that the titles designating appointments of various grades vary from hospital to hospital. Specifically, the term Resident in some centres indicates a senior house appointment, available only after considerable training in Anaesthesia, while in other centres and individual hospitals it is a term applied to all postgraduate interns-in-training. The terminology used in each case below is that in use by the hospital or university in question, and the exact nature of each appointment must be deduced by reference to the qualifications required for it

General Hospital, St John's

NEWFOUNDLAND

\begin{tabular}{lcccccc}
\hline Appointments & Number & Duration & $\begin{array}{c}\text { Avalable } \\
1958\end{array}$ & Remuneration & Requirements \\
\hline $\begin{array}{l}\text { Resident } \\
\text { Anaesthetist }\end{array}$ & 3 & $1 \mathrm{yr}$ & $1-$ Jan & $\begin{array}{c}\$ 3,60000 \text { less } \$ 66000 \\
\text { 1f living in }\end{array}$ & $\begin{array}{c}\text { Previouts experience } \\
\text { 1n Anaesthesia de- } \\
\text { sirable but not } \\
\text { essential }\end{array}$ \\
\hline
\end{tabular}

Basic sczence tramnng There are no facilities for teaching basic sciences Presentation of papers and informal discussions occur at regular departmental meetings

Address applications to Dr E Wilson,

Superintendent, General Hospital,

$\mathrm{St}$ John's, Newfoundland

Dalhousie University, Halifax

NOVA SCOTIA

Teaching hospitals Victoria General Hospital

Children's Hospital

Camp Hill Hospital (D V A)

\begin{tabular}{lcccccc}
\hline Appointments & Number & Duration & $\begin{array}{c}\text { Available } \\
1958\end{array}$ & Remuneration & Requirements \\
\hline Senior Intern & 3 & $1 \mathrm{yr}$ & 0 & $\$ 10000 / \mathrm{mo}$ & $\begin{array}{c}\text { Graduate of Class A } \\
\text { Medical School plus } \\
\text { one year general } \\
\text { internship }\end{array}$ \\
$\begin{array}{c}\text { Resident } \\
\text { Anaesthetist }\end{array}$ & 3 & $1 \mathrm{yr}$ & 2 & $\$ 12500 / \mathrm{mo}$ & $\begin{array}{c}\text { Residents are re- } \\
\text { apponted yearly } \\
\text { for three years }\end{array}$ \\
Senior Resident & 1 & $1 \mathrm{yr}$ & 1 & $\$ 15000 /$ mo & \\
\hline
\end{tabular}

Basuc scuence traınung One year in Medicine or Basic Sciences avallable Anatomy--one afternoon per week for six months, Pharmacology-two residents as demonstrators in the department, lectures by physiologist and members of the department of Anaesthesia Residents are asked to attend suitable meetings of other services in the hospital and lectures given by outstanding visiting doctors to numerous refresher courses Residents and interns are rotated through the Children's Hospital, the D V A Hospital for periods of two to three months and to the Naval Hospital on a darly basis

Address applications to Dr Carl Stoddard,

Victoria General Hospital,

Halıfax, Nova Scotia 
Saint John General Hospital

NEW BRUNSWICK

\begin{tabular}{lccccc}
\hline \hline Appointments & Number & Duration & $\begin{array}{c}\text { Avalable } \\
1958\end{array}$ & Remuneration & Requirements \\
\hline Sentor Intern & 1 & $1 \mathrm{yr}$ & 1 & $\begin{array}{c}\$ 7500 / \text { mo plus } \\
\$ 25.00 \text { living out } \\
\text { allowance }\end{array}$ & Graduate of one year \\
\hline
\end{tabular}

Basuc scrence tramung Clinical teaching only

Address applucations to Dr Carl R Trask,

Director,

Saint Jobn General Hospital,

Sant John, New Brunswick

Laval University, QUeBEC

QUEBEC

Teachıng hospitals Hôtel-Dieu DE QuéBEc

Lival Hospital, Ste Foy, Québec

Hôtel-Dueu de Québec

\begin{tabular}{|c|c|c|c|c|c|}
\hline Appointments & Number & Duration & $\begin{array}{c}\text { Avaulable } \\
1958\end{array}$ & Remuneration & Requirements \\
\hline \multirow{3}{*}{$\begin{array}{l}\text { Senior Intern } \\
\text { Resident } \\
\text { Anaesthetist } \\
\text { Research Fellow }\end{array}$} & $\underline{0}$ & $1 \mathrm{yr}$ & 0 & $\$ 10000 / \mathrm{mo}$ & \\
\hline & 7 & 3 yrs & 0 & $\$ 20000 / \mathrm{mo}$ to & \\
\hline & 0 & - & 1 & - & \\
\hline \multirow{2}{*}{\multicolumn{6}{|c|}{$\begin{aligned} & \text { Basic science training Not specified Senior inte } \\
& \text { 1ddress applications to } \text { Dr Fernando Hudon, } \\
& \text { Hôtel-Dieu Hospital, } \\
& \text { Quebec, PQ }\end{aligned}$}} \\
\hline & & & & & \\
\hline \multicolumn{6}{|c|}{ Laval Hosputal, Ste Foy } \\
\hline Ippointments & Number & Duration & $\begin{array}{c}\text { Avallable } \\
1958\end{array}$ & Remuneration & Requirements \\
\hline $\begin{array}{l}\text { Resident } \\
\text { Anaesthetist }\end{array}$ & 2 & $1 \mathrm{yr}$ & 2 & $\begin{array}{l}\$ 3,00000 \text { per } \\
\text { annum }\end{array}$ & $\begin{array}{l}\text { Two years trainıng } \\
\text { (minımum) }\end{array}$ \\
\hline
\end{tabular}

Basic science training As provided by Laval University

Address applications to ,Dr A L'Espérance,

Medical Director,

Laval Hospital,

Ste Foy, Quebec

Hôpital de L'Enfant-JÉsus, Quebec

\begin{tabular}{|c|c|c|c|c|c|}
\hline Appointments & Number & Duration & $\begin{array}{c}\text { Available } \\
1958\end{array}$ & Remuneration & Requirements \\
\hline Senor Intern & 1 & $1 \mathrm{yr}$ & 1 & $\$ 10000 / \mathrm{mo}$ & $\begin{array}{l}\text { One year rotating } \\
\text { internship, one year } \\
\text { senior internship }\end{array}$ \\
\hline $\begin{array}{l}\text { Resident } \\
\text { Anaesthetist }\end{array}$ & 4 & 3 yro & 1 & $\begin{array}{l}\$ 10000 / \mathrm{mo} \\
\text { to } \$ 30000 / \mathrm{mo}\end{array}$ & \\
\hline $\begin{array}{l}\text { Clinical } \\
\text { Assistant }\end{array}$ & 3 & - & 0 & - & $\begin{array}{l}\text { Residency of three } \\
\text { years }\end{array}$ \\
\hline
\end{tabular}

Basuc scrence traznng Weekly theoretical courses and instructions from staff members each morning Teaching is in French

Address applications to DI Eugene Allard,

Chref, Department of Anaesthesia,

Hôpital de l'Enfant-Jésus,

Quebec, $\mathrm{PQ}$ 
Hópital St. Francis d'Assise, QUebec

\begin{tabular}{|c|c|c|c|c|c|}
\hline Appointments & Number & Duration & $\begin{array}{c}\text { Available } \\
1958\end{array}$ & Remuneration & Requirements \\
\hline $\begin{array}{l}\text { Senior Intern } \\
\text { Resident } \\
\text { Anaesthetist }\end{array}$ & $\begin{array}{l}2 \\
2\end{array}$ & $\begin{array}{l}4 \text { mo } \\
3 \text { yrs. }\end{array}$ & $\begin{array}{l}1 \\
2\end{array}$ & $\begin{array}{l}\$ 7500 / \mathrm{mo} \text {. } \\
\$ 20000 / \mathrm{mo}\end{array}$ & \\
\hline $\begin{array}{l}\text { Clinical Assistant } \\
\text { Part-time }\end{array}$ & $\begin{array}{l}1 \\
2\end{array}$ & $\begin{array}{c}3 \text { yrs } \\
\text { Indefinite }\end{array}$ & $\begin{array}{l}1 \\
0\end{array}$ & $\begin{array}{l}\$ 50000 / \mathrm{mo} \\
\$ 20000 / \mathrm{mo}\end{array}$ & \\
\hline
\end{tabular}

Four years training is acceptable by the College of Physicians of Province of Quebec, one year by the Royal College of Physicians and Surgeons of Canada

Basic science trannng Four hours each week of basic training from September to June

Address applications to Dr Roland Duchesne,

Director of Anaesthesia,

Hôpital St Francis D'Assise,

Quebec, $\mathrm{P} Q$

Hôpital Genteral St Vincent de PaUl, Sherbrooke

\begin{tabular}{lccccc}
\hline Appointments & Number & Duration & $\begin{array}{c}\text { Avallable } \\
1958\end{array}$ & Remuneration & Requirements \\
\hline $\begin{array}{l}\text { Resident } \\
\text { Anaesthetist }\end{array}$ & 1 & $1 \mathrm{yr}$ & 1 & $\$ 12500 / \mathrm{mo}$ & $\begin{array}{c}\text { Graduate of approved } \\
\text { Medical School }\end{array}$ \\
\hline
\end{tabular}

Basuc science tramng Not specified

Address applicatzons to. Dr Fernand Tanguay,

Medical Director

Hôpital Général St Vincent de Paul,

Sherbrooke, $\mathrm{PQ}$

General Hospital, Verdun

\begin{tabular}{lccccc}
\hline \hline Appointments & Number & Duration & $\begin{array}{c}\text { Avalable } \\
1958\end{array}$ & Remuneration & Requirements \\
\hline $\begin{array}{l}\text { Resident } \\
\text { Anaesthetist }\end{array}$ & 1 & $1 \mathrm{yr}$ & 1 & $\$ 40000 / \mathrm{mo}$ \\
\hline
\end{tabular}

Basu scrence trammg Not specified

Address applucations to Anaesthesia Service,

200 Church St,

Verdun, $P Q$ 
MCGill University, Montreal

Teaching hospitals-M Montreal General Hospital

Royal Victoria Hospital, Montreal

MONTREAL NEUROLOGICAL InSTITUTE

Children's Memorial Hospital, Montreal

St Mary's Hospital, Montreal

QueEn Mary Veteran's Hospital, Montreal

QueEN Elizabeth Hospital, Montreal

RedDy Memorial Hospital, Montreal

Royal Edward LaUrentian Hospital, Montreal.

\begin{tabular}{|c|c|c|c|c|c|}
\hline Appointments & Number & Duration & $\begin{array}{c}\text { Avarlable } \\
1958\end{array}$ & Remuneration & Requirements \\
\hline $\begin{array}{l}\text { Resident in } \\
\text { Anaesthesia }\end{array}$ & 36 & 8 yrs & $\begin{array}{l}\text { Some } \\
\text { July } 1\end{array}$ & $\begin{array}{l}\text { 1st yr } \$ 10000 / \mathrm{mo} \\
\text { 2nd yr } \$ 15000 / \mathrm{mo} \\
\text { 3rd yr } \$ 15000 / \mathrm{mo} \text {. } \\
\text { plus either an } \\
\text { allowance or full } \\
\text { maintenance }\end{array}$ & Rotating internship \\
\hline
\end{tabular}

The course is three years, leading to a Diploma in Anaesthesia from $\mathrm{McG}_{\mathrm{c}} 1 \mathrm{ll}$ University Students are assigned for clinical residency to the co-operating hospitals on a six-month rotation basis

Basic science traming Organized courses in Anatomy, Physiology, Biochemistry, and Pharmacology are conducted by the various university departments Two-hour seminars are held two evening a week throughout the teaching session of thirty weeks

Research The Wellcome Founda1 ion Anaesthesia Research Department of McGill which is under the direction of Professor Gordon Robson enables postgraduate students to study methods of research in Anaesthesia Those postgraduate students specially interested in learning or undertaking projects in this field may write to Professor Gordon Robson direct

For short-term residency in the above hospitals apply directly to the Head of the Department of Anaesthesia of that particular hospital

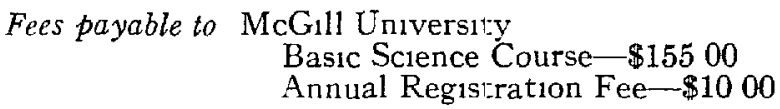

Address applzcatqons to $\mathrm{R} \mathrm{G} B$ Gilbert, $\mathrm{M} \mathrm{B}$,

Charma of the Department of Anaesthesia)

McGill University,

Montrea! Neurological Institute,

Montreal 2, P Q

Montreal Chuldren's Hosputal and Reddy Memorzal Hospital, Montreal

Senzor residency appointments are avallable for up to two years' recognized training, predominantly paediatric The ma ority of the junior resident staff are obtaned as part of the McGill diploma trainıng program $F$ owever, independent inquirıes are welcome, and those interested in obtaining special experience in paediatrics can usually be catered for if they will apply directly to.

Dr $\mathrm{H}$ T Davenport,

Director of Anaesthesia,

Montreal Childi en's Hospital,

2300 Tupper St ,

Montreal, P Q 
Royal Victoria Hospital, Montreal

\begin{tabular}{|c|c|c|c|c|c|}
\hline Appointments & Number & Duration & $\begin{array}{c}\text { Avallable } \\
1958\end{array}$ & Remuneration & Requirements \\
\hline $\begin{array}{l}\text { Assistant } \\
\text { Residents }\end{array}$ & 5 & $6-12 \mathrm{mo}$ & 5 & $\begin{array}{l}\$ 10000 / \mathrm{mo} \text { plus } \\
\$ 4000 / \mathrm{mo} \text { living } \\
\text { out allowance }\end{array}$ & $\begin{array}{l}\text { Approved junior } \\
\text { rotating internship }\end{array}$ \\
\hline $\begin{array}{l}\text { Assistant } \\
\text { Residents }\end{array}$ & 4 & $6-12 \mathrm{mo}$ & 4 & $\begin{array}{l}\$ 15000 / \text { mo plus } \\
\$ 4000 / \text { mo living } \\
\text { out allowasce }\end{array}$ & $\begin{array}{l}\text { One year postgraduate } \\
\text { tratning in } \\
\text { anaesthesia }\end{array}$ \\
\hline $\begin{array}{l}\text { Resident in } \\
\text { Anaesthesia }\end{array}$ & 3 & $6-12 \mathrm{mo}$ & 3 & $\begin{array}{l}\$ 20000 / \mathrm{mo} \text { plus } \\
\$ 4000 / \mathrm{mo} \text { living } \\
\text { out allowance }\end{array}$ & $\begin{array}{l}\text { Two years post- } \\
\text { graduate trainıng } \\
\text { in allaesthesia }\end{array}$ \\
\hline Clinical Fellow & 4 & $1 \mathrm{yr}$ & $\begin{array}{l}2-\text { an } 1 \\
2-\text { uly }\end{array}$ & $\$ 60000 / \mathrm{mo}$ & $\begin{array}{l}\text { These appointments } \\
\text { available to students } \\
\text { of the final year of } \\
\text { supervised practice, } \\
\text { required by the } \\
R \text { C.P S of Canada } \\
\text { for Certification }\end{array}$ \\
\hline
\end{tabular}

Basuc science traming As a participating hospital in the McGill Diploma Course in Anaesthesia, all members of the resident staff are encouraged to register as postgraduate students at $\mathrm{McG}_{1} 11$
Addiess applications fol resident appointments to Dr J Gilbert Turner, Executive Director, Royal Victoria Hospital. Montreal 2, $\mathrm{PQ}$
Address applications for Clinucal Fellowshops to Dr A B Noble; Anaesthetist-1n-Chief, Royal Victoria Hospital, Montreal 2, PQ

INIVERSITY OF VONIREAL, MONTRHAL.

leaching horpitals Hôpital Maisonneuvt, HôpITal Notre-Dame, HôtEl-DiEt DE MONTREAL

\begin{tabular}{|c|c|c|c|c|c|}
\hline Appointments & Number & Duration & $\begin{array}{c}\text { travlable } \\
1958\end{array}$ & Remuneration & Requirementu \\
\hline Senior Intern & 10 & & 12 & $\$ 9000 / \mathrm{mo}$ & \\
\hline $\begin{array}{l}\text { Resident } \\
\text { tnaesthetist }\end{array}$ & 10 & & 12 & $\$ 25000 / \mathrm{mo}$ & \\
\hline Clinical Assistant & 5 & & 10 & $\$ 30000 / \mathrm{mo}$ & \\
\hline
\end{tabular}

Basıc sczence hatming Pharmacology-12 lectures, B ochemistry-12 lectures, Anatomy - 12 lectures, Physiology- 12 lecturen, and clinical application in different hospitals

Address applicatrons to Dr Léon Longtun, Enuercity of Montrcal, Vontreal, PQ 
Matsonneuve Hosputal (\& Instutute of Carduology)

\begin{tabular}{|c|c|c|c|c|c|}
\hline Appontments & Number & Duration & $\begin{array}{c}\text { Avarlable } \\
1958\end{array}$ & Remuneration & Requirements \\
\hline Senır Intern & ] & $1 \mathrm{yr}$ & 1 & $\begin{array}{l}\$ 20000 \text { to } \\
\$ 25000 / \mathrm{mo} .\end{array}$ & $\begin{array}{l}\text { One year rotating } \\
\text { internship }\end{array}$ \\
\hline $\begin{array}{l}\text { Resident } \\
\text { Inaesthetrist }\end{array}$ & 2 & yr & 2 & $\begin{array}{l}\$ 30000 \text { to } \\
\$ 35000 / \mathrm{mo}\end{array}$ & $\begin{array}{l}\text { Rotation plus one } \\
\text { year senior intern- } \\
\text { ship in Anaesthesid }\end{array}$ \\
\hline $\begin{array}{l}\text { Clinical } \\
\text { Assistant }\end{array}$ & 4 & l vr & 3 & $\begin{array}{l}\$ 40000 \text { to } \\
\$ 45000 / \mathrm{mo}\end{array}$ & $\begin{array}{l}\text { Two years' training } \\
\text { in Anaesthesia }\end{array}$ \\
\hline Clinical Fellow & 3 & 6 nio plus & 1 & $\$ 50000 /$ mo up & $\begin{array}{l}\text { Three years' trainung } \\
\text { in Anaesthesia } \\
\text { (pending certifi- } \\
\text { cation or newly } \\
\text { certified) }\end{array}$ \\
\hline
\end{tabular}

Basic science training As provided by Diploma Cołrse at University of Montreal or McGill ['niversity

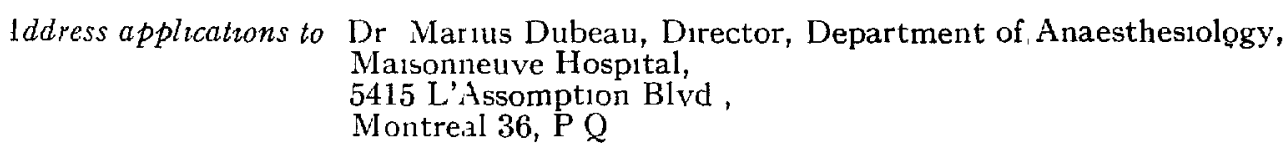

University OF 'Toronto, Toronto

\section{ONTARIO .}

Teachung hospitals Toronto General Hospital

TORONTO Western Hospital

'St Michael's Hospital, Toronto

Hospital for Sick Children, TORONTo

Toronto East General and Orthopaedrc Hospital

WOMEN'S COllege Hospital, TORONTO

SUNNYBrook Hospital (D V A), TORONTO

New Mount Sinai Hospital, Toronto

Hamilton General Hospital, Hamilton

\begin{tabular}{|c|c|c|c|c|c|}
\hline Appointments & Nứnber & Duration & $\begin{array}{c}\text { Ava.1lable } \\
1958\end{array}$ & Remuneration & Requirements \\
\hline Senior Intern & 21 & 2 yrs & 10 & $\begin{array}{c}\$ 1,00000 \text { to } \\
\$ 1,40000\end{array}$ & $\begin{array}{l}\text { (1) Qualification } \\
\text { registrable to } \\
\text { practise medicine } \\
\text { in Ontario } \\
\text { (2) One year rotating } \\
\text { 1nternship }\end{array}$ \\
\hline $\begin{array}{l}\text { Resident } \\
\text { Anaesthetist }\end{array}$ & 5 & $1 y$ & 5 & $\begin{array}{l}\$ 1,80000 \text { plus } \\
\$ 30000 \text { from } \\
\text { University }\end{array}$ & $\begin{array}{l}\text { As above plus two } \\
\text { years' trainıng } 111 \\
\text { Anaesthesia }\end{array}$ \\
\hline $\begin{array}{l}\text { Clunical } \\
\text { Assistant }\end{array}$ & 2 & $1 \mathrm{yr}$ & 1 & - & $\begin{array}{l}\text { As for Resident } \\
\text { Anaesthetist plus } \\
\text { one year in Internal } \\
\text { Medicine or a } \\
\text { Basic Science }\end{array}$ \\
\hline $\begin{array}{l}\text { Resedrch } \\
\text { Fellow }\end{array}$ & 2 & $1 \mathrm{yr}$ & 1 & $\$ 2,000.00$ & $\begin{array}{l}\text { As for Resident } \\
\text { Anaesthetist plus } \\
\text { some experience in } \\
\text { research in a basic } \\
\text { science }\end{array}$ \\
\hline
\end{tabular}

Basic scuence training Anatomy-32 one and a half hour lecture demonstrations in Department of Anatomy, Pathology - lecture of one and a half.hour a week for eight months a year, Physiology - lectures and seminars during two-year period from Sept to May, Pharmacology-lectures and heminars during two-year period from Sept to May

Addiess applicalions to Dr S M Campbell, Associate Professor, Department of Anaesthesia, Iniversity of Toronto 
ST. Joseph's Hospital, ToRonto

\begin{tabular}{|c|c|c|c|c|c|}
\hline Appointments & Number & Duration & $\begin{array}{c}\text { Avallable } \\
1958\end{array}$ & Remuneration & Requirements \\
\hline Senior Intern & 1 & $1 \mathrm{yr}$. & 0 & $\$ 1,700.00$ & 1 yr. rotation \\
\hline $\begin{array}{l}\text { Resident } \\
\text { Anaesthetist }\end{array}$ & 1 & $1 \mathrm{yr}$ & 0 & $\$ 3,000.00$ & $\begin{array}{l}2 \text { yrs training in } \\
\text { anaesthesia }\end{array}$ \\
\hline Other (Junior) & & $1 \mathrm{mo}$ & 1 & $\$ 1,400.00$ & $\begin{array}{l}\text { Graduate of recognized } \\
\text { medical school }\end{array}$ \\
\hline
\end{tabular}

Basic science training None

Address applacations to . Sister Superior,

St Joseph's Hospital,

Toronto, Ontario

QUeen's University, Kingston

Teaching hospitals. Kingston GENERAI Hospital,

Hôtel-Dieu Hospital, Kingston

Kingston General Hospital

\begin{tabular}{lcccccc}
\hline Appointments & Number & Duration & $\begin{array}{c}\text { Avallable } \\
1958\end{array}$ & Remuneration & Requirements \\
\hline $\begin{array}{l}\text { Senior Intern } \\
\begin{array}{l}\text { Resident } \\
\text { Anaesthetıst }\end{array}\end{array}$ & 1 & $1 \mathrm{yr}$ & 1 & $\$ 20000 / \mathrm{mo}$ & $\begin{array}{c}\text { Junior rotating } \\
\text { internship }\end{array}$ \\
& $1 \mathrm{yr}$. & 1 & $\$ 25000 / \mathrm{mo}$ & $\begin{array}{c}\text { Junior rotating } \\
\text { internship plus } \\
\text { one year training } \\
\text { in Anaesthesia }\end{array}$ \\
\hline
\end{tabular}

Basic scuence training As arranged with individual university departments

Address appincations to $\mathrm{Dr} W$ A. Campbell,

Chief, Department of Anaesthesia,

Kingston General Hospital,

Kingston, Ontarı

Hôtel-Dieu Hosputal

\begin{tabular}{lccccc}
\hline Appointments & Number & Duration & $\begin{array}{c}\text { Avalable } \\
1958\end{array}$ & Remuneration & Requirements \\
\hline Senior Intern & 2 & $1 \mathrm{yr}$ & 2 & $\$ 20000 /$ mo & $\begin{array}{c}\text { One year rotating } \\
\text { internship }\end{array}$ \\
\hline
\end{tabular}

Baszc scrence traning Weekly seminars, covering physılogy, pharmacology, anatomy and biochemistry and their application in Anaesthesia

Address applications to: Dr. V. A. Nekus,

Department of Anaesthesia,

Hôtel-Dieu Hospital,

Kingston, Ontario 
UNIVERSÍTY OF WESTERN ONTARIO, LONDON

Teaching hospitals: VICTORIA HosPITAL, LONDON

ST JOSEPH's HOSPITAL, LONDON

\begin{tabular}{|c|c|c|c|c|c|}
\hline Appointments & Number & Duration & $\begin{array}{c}\text { Available } \\
1958\end{array}$ & Remuneration & Requirements \\
\hline \multirow[t]{2}{*}{ Sensor Intern } & 1 & 2 yrs. & Jan. $1 / 58-$ & $\$ 150.00 / \mathrm{mo}$ & Junior rotating \\
\hline & 2 & 2 yrs. & $\begin{array}{l}\text { July 1/58- } \\
\text { July } 1 / 59\end{array}$ & $\$ 125.00 / \mathrm{mo}$ & L.M.C.C. \\
\hline $\begin{array}{l}\text { Resıdent } \\
\text { Anaesthetist }\end{array}$ & 1 & $1 \mathrm{yr}$. & $\begin{array}{c}\text { Jan. } 1 / 58 \\
\text { Jan. } 1 / 59\end{array}$ & $\$ 150.00 / \mathrm{mo}$ & $\begin{array}{l}\text { Two years as senior } \\
\text { plus L.M.C.C. }\end{array}$ \\
\hline
\end{tabular}

Basuc scuence tramnng Senior and Resident interns are required to spend part time at the Medical School as Research Assistants during their internships in addition to their hospital work Grants are usually avalable from which additional remuneration is received.

Address applications to: Dr. J. A. Blezard

Head of Department of Anaesthesia,

Unversity of Western Ontario,

London, Ontario

Westmınster Hospital (D.V A), London

\begin{tabular}{lcccccc}
\hline Appointments & Number & Duration & $\begin{array}{c}\text { Available } \\
1958\end{array}$ & Remuneration & Requirements \\
\hline $\begin{array}{l}\text { Assistant } \\
\text { Resident }\end{array}$ & 1 & $1 \mathrm{yr}$ & 1 & $\$ 2,700.00$ & $\begin{array}{c}\text { Two years acceptable } \\
\text { postgraduate train- } \\
\text { ing, not necessarily } \\
\text { in Anaesthesia }\end{array}$ \\
\hline
\end{tabular}

Basic science training. Not specified.

Address applicatrons to: Dr. William Bugg,

Assistant Medical Superintendent,

Westminster Hospital,

London, Ontario

Ottawa Civic Hosputai

\begin{tabular}{lcccccc}
\hline \hline Appointments & Number & Duration & $\begin{array}{c}\text { Available } \\
1958\end{array}$ & Remuneration & Requirements \\
\hline $\begin{array}{l}\text { Resident } \\
\text { Anaesthetıst }\end{array}$ & 3 & $1 \mathrm{gr}$. & 3 & $\begin{array}{c}\$ 20000 / \text { mo. plus } \\
\text { full maintenance }\end{array}$ & $\begin{array}{c}\text { One year rotating } \\
\text { internship }\end{array}$ \\
\hline
\end{tabular}

Baszc sczence training A series of basic science lectures, organized by the Post-Graduate Education Committee, and available to all interns

Address applacahons to: Superintendent,

Ottawa Civic Hospital,

Ottawa, Ontario 
Hamil ron General Hospital, Hamilton

\begin{tabular}{|c|c|c|c|c|c|}
\hline Appointments & Number & Duration & $\begin{array}{c}\text { Avarlable } \\
1958\end{array}$ & Remuneration & Requirements \\
\hline Senior Intern & 2 & $1-6 \mathrm{mo}$ & 1 & $\$ 17500 / \mathrm{mo}$ & \\
\hline $\begin{array}{l}\text { Resident } \\
\text { Anaesthetist }\end{array}$ & 1 & & $1-J u l y$ & & $\begin{array}{l}\text { To be in final year of } \\
\text { postgraduate course } \\
\text { in Anaesthesia }\end{array}$ \\
\hline
\end{tabular}

One senır intern rotating three months on Anaesthesia, the other nine months through Paediatrics, Medicine and Obstetrics At present one senior intern is provided from postgraduate course in Inaesthesia at University of Toronto for a period of sil montha rotation

Basic science training Not specified

Address applications to Medical Assistant to the Superintendent, Hamilton General Hospital, Hamilton, Ontario

St Joseph's Hospital, Hamilton

\begin{tabular}{lccccc}
\hline Appointments & Number & Duration & $\begin{array}{c}\text { Avallable } \\
1958\end{array}$ & Remuneration & Requirements \\
\hline $\begin{array}{l}\text { Resident } \\
\text { Anaesthetist }\end{array}$ & 1 & $1 \mathrm{lr}$ & 1 & $\begin{array}{c}\$ 1500010 \\
\$ 20000 / \text { mo }\end{array}$ & $\begin{array}{c}\text { One year rotating } \\
\text { internship plus } \\
\text { L M C C }\end{array}$
\end{tabular}

Basic science training None Training consists of instruction in inhalation, intrayenous, spinal, regional and rectal anaesthetic techniques Weekly review of literature presented to staff, and bi-annually to section of Anaesthesia of Academy of Medicine

$\begin{aligned} \text { Address applications to } & \text { Dr Karl Kraft, } \\ & \text { Director of Anaesthesia, } \\ & \text { St Joseph's Hospital, } \\ & \text { Hamilton, Ontario }\end{aligned}$

\section{MIANIIOBA}

St Boniface Hospital, St Boniface

\begin{tabular}{|c|c|c|c|c|c|}
\hline Appointments & Number & Duration & $\begin{array}{c}\text { Avaılable } \\
1958\end{array}$ & Remuneration & Requirements \\
\hline Senior Intern & 2 & $1 y T$ & 2 & $\$ 22500 / \mathrm{mo}$ & $\begin{array}{l}\text { Graduate from Grade } \\
\text { I Medical School } \\
\text { plus one year } \\
\text { rotating internship }\end{array}$ \\
\hline $\begin{array}{l}\text { Resident } \\
\text { Anaesthetist }\end{array}$ & 2 & $1\} r$ & 0 & $\$ 35000 / \mathrm{mo}$ & $\begin{array}{l}\text { As above plus one } \\
\text { year senior intern } \\
\text { in Anaesthesia }\end{array}$ \\
\hline $\begin{array}{l}\text { Clinical } \\
\text { Assistant }\end{array}$ & 1 & 1 Ir & 0 & & \\
\hline Clinical Fellow & 1 & $1 \mathrm{yr}$ & 0 & & \\
\hline
\end{tabular}

Basuc science tramıng Yearly course of lectures by the Manitoba Medical College The course includes lectures in Anatomy, Physiology, Biochemistry and Pharmacology

Address applications to Dr M Minuck,

St Bonfface Hospital,

St Bonface, Mantobal 
DeEr Lodge Hospital (D V A.), Winnipeg

\begin{tabular}{lcccccc}
\hline \hline Appointments & Number & Duration & $\begin{array}{c}\text { Avallable } \\
1958\end{array}$ & Remuneration & Requirements \\
\hline Senior Intern & 1 & 2 yrs & 0 & $\$ 250 \div 00 /$ mo & $\begin{array}{c}\text { One year rotating } \\
\text { internship }\end{array}$ \\
\hline
\end{tabular}

Bası science traming Weekly lectures in Basic Sciences in conjunction with the University of Manitoba postgraduate course

Address applications to Director of Department of Anaesthesia,

Deer Lodge Hospital,

Winnipeg 12, Manitoba

Winnipeg General Hospital, Winnipeg

\begin{tabular}{|c|c|c|c|c|c|}
\hline Appointments & Number & Duration & $\begin{array}{c}\text { Avaılable } \\
1958\end{array}$ & Remuneration & Requirements \\
\hline Senior Intern & 2 & l yr & 0 & $\begin{array}{l}\$ 90000 \text { plus board } \\
\text { and room }\end{array}$ & \\
\hline $\begin{array}{l}\text { Assistant } \\
\text { Resident }\end{array}$ & 1 & L yr & 1 & $\begin{array}{l}\$ 1,50000 \text { to } \\
\$ 1,80000\end{array}$ & \\
\hline $\begin{array}{l}\text { Resident } \\
\text { Anaesthetist }\end{array}$ & 1 & lyr & 1 & $\begin{array}{l}\$ 2,10000 \text { to } \$ 2,700,00 \\
\text { plus board and room }\end{array}$ & \\
\hline $\begin{array}{l}\text { Clinical } \\
\text { Assistant }\end{array}$ & 1 & L yr & 1 & $\$ 4,00000$ & \\
\hline
\end{tabular}

Basuc scuence tramung Training is provided through the Basic Science Departments of the $L^{\top} n 1-$ versity of Manitoba

Address applications to Dr D U Huggins, Department of Anaesthesia, Winnipeg General Hospital, Winnipeg, Manitoba

Salvation Army Grace Hospital, Winnipeg

\begin{tabular}{lccccc}
\hline Appointments & Number & Duration & $\begin{array}{c}\text { Avalable } \\
1958\end{array}$ & Remuneration & Requirements \\
\hline Resident & 1 & $6 \mathrm{mo}$ & 1 & $\$ 25000 / \mathrm{mo}$ & \\
\hline
\end{tabular}

Basic scuence training Universıy of Manitoba postgraduate training programme in Anaesthesia Address applications to $\mathrm{Dr} \mathrm{S} \mathrm{M}$ Luginsky, Salvation Army Grace Hospital, 200 Arlington St, Winnıpeg, Manitoba

Childoren's Hospital, Winnipeg

\begin{tabular}{lccccc}
\hline \hline Appointments & Number & Duration & $\begin{array}{c}\text { Available } \\
1958\end{array}$ & Remuneration & Requirements \\
\hline $\begin{array}{l}\text { Clinical } \\
\text { Assistant }\end{array}$ & 1 & $1 \mathrm{yr}$ & 1 & $\begin{array}{c}\text { Not more than } \\
\$ 40000 \text { per mo }\end{array}$ & $\begin{array}{c}2 \text { yrs "basic" training } \\
\text { in Anaesthesia in } \\
\text { a recognized hospital } \\
\text { taken previously }\end{array}$ \\
\hline
\end{tabular}

Basuc scuence traınıng Post-Graduate Course in Anaesthesı, Unıversity of Manıtoba.

Address applications to Dr I J McCaughey,

Director, Dept of Anaesthesia,

Children's Hospital,

Winnıpeg, Manitoba 


\section{SASKATCHEWAN}

UNIVERSity of SaSkatchewan, SASKatoon

Teaching hospitals University Hospital, SASKatioOn Saskatoon SaNatorium

\begin{tabular}{lccccc}
\hline Appointments & Number & Duration & $\begin{array}{c}\text { Available } \\
1958\end{array}$ & Remuneration & Requirements \\
\hline Senior Intern & 2 & $1 \mathrm{yr}$ & 2 & $\$ 150.00 / \mathrm{mo}$ & $\begin{array}{c}\text { Rotating internship if } \\
\text { practising in } \\
\text { Canada, internship } \\
\text { not required if alien } \\
\text { returning home after } \\
\text { training }\end{array}$ \\
$\begin{array}{c}\text { Resident } \\
\text { Anaesthetist }\end{array}$ & 3 & $2 \mathrm{yrs}$ & 3 & $\begin{array}{c}\$ 20000 / \text { mo to } \\
\$ 22500 / \mathrm{mo}\end{array}$ & $\begin{array}{c}\text { Senior internship in } \\
\text { Anaesthesia or } \\
\text { Medicine }\end{array}$ \\
$\begin{array}{l}\text { Research Fellow } \\
\text { Full training, } \\
\text { preferably holding } \\
\text { higher qualification }\end{array}$ \\
\hline
\end{tabular}

Basic sczence training A full range of seminars (3 per week) is provided covering all basic sciences as related to Anaesthesia Some of these semmars are given by members of the Departments of Inatomy, Physiology, and Pharmacology. Test-papers are given from time to time as certain aspects of basic science fields are completed

Address apphcatzons to $\mathrm{Dr}$ Gordon M Wyant,

Professor of Anaesthesia,

University of Saskatchewan,

Saskatoon, Saskatchewan

Regina General Hospital, Regina

\begin{tabular}{lccccc}
\hline \hline Appointments & Number & Duration & $\begin{array}{c}\text { Avallable } \\
1958\end{array}$ & Remuneration & Requirements \\
\hline $\begin{array}{l}\text { Resident } \\
\text { Anaesthetıst }\end{array}$ & 2 & 1 yr & 1 & $\$ 25000 /$ mo & $\begin{array}{c}1 \text { year rotating } \\
\text { internship }\end{array}$ \\
\hline
\end{tabular}

Address applucatnons to. The Superintendent, Regina General Hospital, Regina, Saskatchewan 
ALBERTA

UNIVERSITY OF AlBERTA, EDMONTON

Teaching hospitals UNIVERSITy Hospital, EDMonton

Edmonton General Hospital,

Royal ALEXANDra Hospital, EDMONTON

Unversily Hosputal, Edmonton

\begin{tabular}{|c|c|c|c|c|c|}
\hline Appointments & Number & Duration & $\begin{array}{c}\text { Avallable } \\
1958\end{array}$ & Remuneration & Requirements \\
\hline Senior Intern & 2 & 2 or 3 yrs & 1 & $\begin{array}{l}\$ 10000 / \mathrm{mo} \text { to } \\
\$ 12500 / \mathrm{mo} \text {. }\end{array}$ & $\begin{array}{l}\text { One year rotating } \\
\text { internship }\end{array}$ \\
\hline $\begin{array}{l}\text { Resident } \\
\text { Anaesthetist }\end{array}$ & 2 & 1 or 2 yrs & 1 & $\$ 15000 / \mathrm{mo}$ & $\begin{array}{l}\text { As above plus one } \\
\text { year in Anaesthesia }\end{array}$ \\
\hline $\begin{array}{l}\text { Chinical } \\
\text { Assistant }\end{array}$ & 2 & $1 \mathrm{yr}$ & 0 & $\begin{array}{l}\text { Variable on } \\
\text { percentage }\end{array}$ & $\begin{array}{l}\text { Eligible for } \\
\text { certification }\end{array}$ \\
\hline Research Fellow & 2 & $1 \mathrm{yr}$ & 2 & $\begin{array}{c}\$ 15000 / \mathrm{mo} \text { to } \\
\$ 200.00 / \mathrm{mo}\end{array}$ & $\begin{array}{l}\text { One year rotating } \\
\text { inteinship }\end{array}$ \\
\hline
\end{tabular}

Bastc scrence trannng Three hours weekly in Laboratory, Pharmacology and Physiology Weekly lectures Research six months to twelve months for Fellowship candidates in Physiology, Pharmacology, Cardiovascular or Pulmonary Laboratories

Address applications to. Intern Commattee,

University of Alberta Hospital,

Edmonton, Alberta

Edmonion General Hospatal

\begin{tabular}{lcccccc}
\hline Appointments & Number & Duration & $\begin{array}{c}\text { Avarlable } \\
1958\end{array}$ & Remuneration & Requirements \\
\hline $\begin{array}{l}\text { Resident } \\
\text { Anaesthetıst }\end{array}$ & 1 & $1 \mathrm{yr}$ & 1 & $\$ 12500 / \mathrm{mo}$ & $\begin{array}{c}\text { As required by Royal } \\
\text { College of } \\
\text { Physicians and } \\
\text { Surgeons of Canada }\end{array}$ \\
\hline
\end{tabular}

Basic sctence trainzng Weekly seminars in conjunction with the Department of Anaesthesia, University of Alberta Hospital Physiology and Pharmacology laboratory work can be arranged through the University of Alberta.

Address applicatzons to. The Medical Director,

Edmonton General Hospital,

Edmonton, Alberta

Calgary General Hospital, Calgary

\begin{tabular}{lcccccc}
\hline \hline Appointments & Number & Duration & $\begin{array}{c}\text { Avalable } \\
1958\end{array}$ & Remuneration & Requirements \\
\hline Senior Intern & 2 & $1 \mathrm{yr}$ & 0 & $\$ 12500 / \mathrm{mo}$ & $\begin{array}{c}\text { One year rotating } \\
\text { internship }\end{array}$ \\
\hline
\end{tabular}

Basic scrence trazning None

Address applacations to: Dr Crosby Johnson, Administrator, Calgary General Hospital, Calgary, Alberta 
University of British Columbia, Vancouver

Teaching hospitals Vancouver General Hospital.

SHAUGHNESSY Veteran's Hospital (D V A ), Vancouvfr

Vancouver General Hospital

\begin{tabular}{|c|c|c|c|c|c|}
\hline Appointments & Number & Duration & $\begin{array}{c}\text { Avallable } \\
1958\end{array}$ & Remuneration & Requirements \\
\hline $\begin{array}{l}\text { Resident } \\
\text { Anaesthetist }\end{array}$ & 2 & & 0 & $\$ 23000 / \mathrm{mo}$ & \\
\hline $\begin{array}{l}\text { Associate } \\
\text { Resident }\end{array}$ & 5 & & 0 & $\$ 20500 / \mathrm{mo}$ & \\
\hline $\begin{array}{l}\text { Assistant } \\
\text { Resident }\end{array}$ & 6 & & 0 & $\$ 16000 / \mathrm{mo}$ & \\
\hline $\begin{array}{l}\text { Clinical } \\
\text { Fellow }\end{array}$ & 1 & & 0 & $\$ 30000 / \mathrm{mo}$ & \\
\hline $\begin{array}{c}\text { Research } \\
\text { Fellow }\end{array}$ & 1 & & 0 & $\$ 200001 / \mathrm{mo}$ & \\
\hline Other & 3 & & 3 & $\$ 20500 / \mathrm{mo}$ & \\
\hline
\end{tabular}

Basıc science traınıng University of Britısh Columbia, Basic Science Department

Address applications to $\mathrm{Dr} \mathrm{H} B$ Graves,

Director,

Department of Anaesthesiology,

Vancouver General Hospital,

Vancouver 9, Britısh Columbıa

Shaughnessy Veteran's Hospital, Vancouver

\begin{tabular}{lccccc}
\hline Appointments & Number & Duration & $\begin{array}{c}\text { Avalable } \\
1958\end{array}$ & Remuneration & Requirements \\
\hline $\begin{array}{l}\text { Senior Intern } \\
\begin{array}{c}\text { Resident } \\
\text { Anaesthetist }\end{array}\end{array}$ & 2 & $1 \mathrm{yr}$ & 2 & $\$ 17500 / \mathrm{mo}$ & $\begin{array}{c}\text { One year junior } \\
\text { Internship }\end{array}$ \\
$\begin{array}{c}\text { Clinical Assistant } \\
\text { (supervised } \\
\text { practice) }\end{array}$ & 1 & $1 \mathrm{yr}$ & 1 & 1 & $\begin{array}{c}\text { Six months to one } \\
\text { year senior intern- } \\
\text { ship in Anaesthesia } \\
\text { During or immediately } \\
\text { following this } \\
\text { position the } \\
\text { incumbent become } \\
\text { eligible for } \\
\text { certification in } \\
\text { Anaesthesia by } \\
\text { examination }\end{array}$ \\
\hline
\end{tabular}

Basic science traınıng Weekly lectures of two hours durıng academic year at Unıversity of Britısh Columbia

Address applications to The Superintendent, Shaughnessy Hospital,

Vancouver 9, British Columbia 
St Paul's Hospital, Vancouver

Detalls not avallable

Address applacahons to $\cdot$ Sister Suprrior,

St Paul's Hospital,

1081 Burrard St

Vancouver 1, Britısh Columbia 